\title{
Enclosure and non-existence theorems for area stationary currents and currents with mean curvature vector
}

\author{
PATRICK HENKEMEYER
}

\begin{abstract}
We discuss certain geometric properties for area stationary currents and currents with integrable mean curvature, so called "enclosure theorems". As a consequence, we obtain non-existence results for currents with connected support. Finally, we extend these results to currents in submanifolds and state a non-existence result for stationary currents in spheres.
\end{abstract}

Mathematics Subject Classification. 49Q15, 49Q20, 53A10, 58A25.

Keywords. Enclosure theorems, Non-existence theorems, Area stationary currents, Currents with mean curvature vectors.

1. Introduction. Let $\Omega \subset \mathbb{R}^{2}$ denote a domain and let $X: \Omega \rightarrow \mathbb{R}^{3}$ be a minimal surface, i.e. a harmonic and conformal mapping of class $C^{2}$. For detailed information on minimal surfaces, we refer to the monographs of Dierkes, Hildebrandt, Sauvigny, and Tromba [4] and [5].

It is well-known that $X(\bar{\Omega})$ is contained in the convex hull of its boundary components $X(\partial \Omega)$. This result holds true for every harmonic mapping due to the maximum principle.

Together with the conformality conditions, Hildebrandt [10] obtained stronger results. In fact, he proved that a minimal surface $X(\bar{\Omega})$ is enclosed by the hyperboloid $H(R):=\left\{(x, y, z) \in \mathbb{R}^{3}: x^{2}+y^{2}-z^{2} \leq R\right\}$ for all $R \in \mathbb{R}$ provided $X(\partial \Omega) \subset H(R)$. For $R=0$, one gets a cone and the theorem still holds true. Furthermore one has that the minimal surface cannot pass through the vertex of the cone. Hildebrandt [10] also stated analogous results for two dimensional $H$-surfaces.

Again, by using the maximum principle for elliptic equations, Dierkes [3] and Dierkes and Schwab [6] extended these results to compact $n$-dimensional $C^{2}$-submanifolds $\mathcal{M}$ in $\mathbb{R}^{n+k}$. By regularity, these results immediately lead 
to non-existence theorems. In fact, there are no smooth connected minimal submanifolds with boundary components in both disjoint parts of a special cone.

The question arises whether this cone can be enlarged or not. For two dimensional minimal surfaces, this was answered by Osserman and Schiffer [13]. They give the optimal "non-existence" cone. Dierkes [3] proved the corresponding theorem for $n$-dimensional smooth submanifolds in $\mathbb{R}^{n+1}$. See also the monograph Dierkes et al. [5, Ch. 4] for a complete survey of these results.

We mention that there are more general results of this type in various situations, cf. [1,9,19], and [2].

Here we want to address the following question: Do these theorems also generalize to currents? We show in the sequel that this is basically the case. The classical maximum principle will be replaced by a maximum principle of Solomon and White [16]. Maximum principles for singular surfaces in general situations were studied by different authors in the last years. We mention $[11,14]$, and recently [18] for codimension 1 and [17] where certain varieties of arbitrary codimension are considered.

We use the notation of Simon [15]. Let $n \geq 2, k \geq 1$ be natural numbers and $U \subset \mathbb{R}^{n+k}$ be an open set. We write $T=\tau(M, \theta, \xi) \in \mathcal{R}_{n}(U)$ for the set of $n$-dimensional rectifiable currents in $U$. Our notation slightly differs from most authors as we do not require integer multiplicity $\theta$. As usual, we define the associated Radon measure $\mu_{T}:=\mathcal{H}^{n}\llcorner\theta$ and $\|\delta T\|$ is the total variation measure. A current with mean curvature $\mathbf{H}=-D_{\mu_{T}}\|\delta T\| \sigma$ is given by

Definition 1.1. Let $T=\tau(M, \theta, \xi) \in \mathcal{R}_{n}(U)$ be a current and $\mathbf{H} \in L_{\text {loc }}^{1}(M \cap$ $\left.U, \mathbb{R}^{n+k} ; \mu_{T}\right)$. Then $T$ has mean curvature vector $\mathbf{H}$ in $U$ if

$$
\int_{U} \operatorname{div}_{M} X \mathrm{~d} \mu_{T}=-\int_{U}\langle\mathbf{H}, X\rangle \mathrm{d} \mu_{T}
$$

whenever $X \in C_{c}^{1}\left(U \backslash \operatorname{spt} \partial T, \mathbb{R}^{n+k}\right)$.

An important special case is $\mathbf{H}(x) \equiv 0$ leading to the definition of "stationary currents" .

\section{Stationary currents.}

2.1. Enclosure theorem. The following convex hull property for stationary currents is well-known, cf. [15, Thm. 19.2, Rmk. 34.2].

Theorem 2.1. Let $T \in \mathcal{R}_{n}\left(\mathbb{R}^{n+k}\right)$ be a stationary current in $\mathbb{R}^{n+k}$ with compact support. Then $\operatorname{spt} T \subset \operatorname{conv}(\operatorname{spt} \partial T)$.

We prove an enclosure result in non-convex sets. Therefore we define for $j=1, \ldots, n-1, r_{j}(x):=\sum_{i=1}^{n+k-j} x_{i}^{2}$ and $s_{j}(x):=\sum_{i=n+k-j+1}^{n+k} x_{i}^{2}$, and the quadratic function $q_{j}(x)=q_{j}\left(x_{1}, \ldots, x_{n+k}\right): \mathbb{R}^{n+k} \rightarrow \mathbb{R}$ given by $q_{j}(x):=$ $r_{j}(x)-\frac{n-j}{j} s_{j}(x)$. Furthermore let $H_{j}(R):=\left\{x \in \mathbb{R}^{n+k}: q_{j}(x) \leq R\right\}$ be a generalized hyperboloid for some $R \in \mathbb{R}$. 
Theorem 2.2 (Enclosure theorem). Let $\mathcal{H}_{j}(R)$ be a generalized hyperboloid for $R \in \mathbb{R}$ and $j=1, \ldots, n-1$ congruent to $H_{j}(R)$. Let $T \in \mathcal{R}_{n}\left(\mathbb{R}^{n+k}\right)$ be a stationary current in $\mathbb{R}^{n+k}$ with compact support spt $T$ and let the boundary values satisfy $\operatorname{spt} \partial T \subset \mathcal{H}_{j}(R)$. Then we have $\operatorname{spt} T \subset \mathcal{H}_{j}(R)$.

Proof. W.l.o.g. assume $\mathcal{H}_{j}(R)=H_{j}(R)$. Let $\varepsilon>0$ be arbitrary and $\gamma \in C^{1}(\mathbb{R})$ be non-negative and non-decreasing with $\gamma(t) \equiv 0, t \leq R+\varepsilon$, and $\gamma(t)>0$, $\gamma^{\prime}(t)>0$ for $t>R+\varepsilon$. Define $\hat{x}: \mathbb{R}^{n+k} \rightarrow \mathbb{R}^{n+k}$ by

$$
\hat{x}(x):=\left(x_{1}, \ldots, x_{n+k-j},-\frac{n-j}{j} x_{n+k-j+1}, \ldots,-\frac{n-j}{j} x_{n+k}\right)
$$

and consider $X(x):=\Psi_{\text {spt } T}(x) \gamma\left(q_{j}(x)\right) \hat{x}(x) \in C_{c}^{1}\left(\mathbb{R}^{n+k} \backslash H_{j}(R), \mathbb{R}^{n+k}\right)$, where $\Psi_{\text {spt } T}(x) \equiv 1$ in a neighborhood of $\operatorname{spt} T$ is a smooth cut-off function.

Let $\mathcal{T}_{x} M$ denote the approximate tangent space of $T$ in $x \in M$ (which exists $\mathcal{H}^{n}$-a.e.) and $\mathcal{P}_{\mathcal{T}_{x} M}: \mathbb{R}^{n+k} \rightarrow \mathcal{T}_{x} M$ the orthogonal projection with matrix representation $\left(p_{i j}\right)_{i, j=1, \ldots, n+k}$ w.r.t. the canonical basis of $\mathbb{R}^{n+k}$. We often abbreviate the projection by $(\cdot)^{\top}$.

For this vector field $X$, we have $\operatorname{div}_{M} X=\left\langle\nabla_{M} \gamma, \hat{x}\right\rangle+\gamma \operatorname{div}_{M} \hat{x}$ and we calculate the different expressions. Firstly, $\nabla_{M} \gamma\left(q_{j}\right)=\gamma^{\prime}\left(q_{j}\right)\left(D q_{j}\right)^{\top}=2 \gamma^{\prime}\left(q_{j}\right) \hat{x}^{\top}$ which gives $\left\langle\nabla_{M} \gamma\left(q_{j}\right), \hat{x}\right\rangle=2 \gamma^{\prime}\left(q_{j}\right)\left\langle\hat{x}^{\top}, \hat{x}\right\rangle=2 \gamma^{\prime}\left(q_{j}\right)\left|\hat{x}^{\top}\right|^{2} \geq 0$ and secondly,

$$
\begin{aligned}
\operatorname{div}_{M} \hat{x} & =\sum_{i=1}^{n+k}\left\langle\nabla_{M} \hat{x}_{i}, e_{i}\right\rangle \\
& =\sum_{i=1}^{n+k-j}\left\langle\nabla_{M} x_{i}, e_{i}\right\rangle-\frac{n-j}{j} \sum_{i=n+k-j+1}^{n+k}\left\langle\nabla_{M} x_{i}, e_{i}\right\rangle \\
& =\sum_{i=1}^{n+k} p_{i i}-\sum_{i=n+k-j+1}^{n+k} p_{i i}-\frac{n-j}{j} \sum_{i=n+k-j+1}^{n+k} p_{i i} \\
& =n-\frac{n}{j} \sum_{i=n+k-j+1}^{n+k} p_{i i} \geq 0 .
\end{aligned}
$$

We have used $\operatorname{tr}\left(\mathcal{P}_{\mathcal{T}_{x} M}\right)=n$ in the last equation. Plugging these into (1.1) yields

$$
0=\int_{\mathbb{R}^{n+k}} \gamma\left(q_{j}\right)\left\{n-\frac{n}{j} \sum_{i=n+k-j+1}^{n+k} p_{i i}\right\}+2 \gamma^{\prime}\left(q_{j}\right)\left|\hat{x}^{\top}\right|^{2} \mathrm{~d} \mu_{T}
$$

For all $i=n+k-j+1, \ldots, n+k$, we have $p_{i i}=1$ iff $e_{i} \in \mathcal{T}_{x} M$. Thus for $\mu_{T}$-a.e. $x \in M$,

$$
\left\{n-\frac{n}{j} \sum_{i=n+k-j+1}^{n+k} p_{i i}\right\}=0 \text { iff } p_{i i}=1 \text { for all } i=n+k-j+1, \ldots, n+k .
$$


Otherwise it is strictly positive. Define the set $E:=\left\{x \in M: \mathcal{T}_{x} M\right.$ exists and $\left.e_{n+k-j+1}, \ldots, e_{n+k} \in \mathcal{T}_{x} M\right\}$. We have on the one hand,

$$
\int_{\mathbb{R}^{n+k} \backslash E} \gamma\left(q_{j}\right)\left\{n-\frac{n}{j} \sum_{i=n+k-j+1}^{n+k} p_{i i}\right\} \mathrm{d} \mu_{T}=0
$$

where the expression $\{\ldots\}$ is positive and due to the definition of $\gamma$, this means spt $\mu_{T} \cap\left(\mathbb{R}^{n+k} \backslash E\right) \subset\left\{x \in \mathbb{R}^{n+k}: q_{j}(x) \leq R+\varepsilon\right\}=H_{j}(R+\varepsilon)$. On the other hand, notice that $\{\ldots\}=0$ in $E$, thus

$$
0=\int_{E} \gamma\left(q_{j}\right)\left\{n-\frac{n}{j} \sum_{i=n+k-j+1}^{n+k} p_{i i}\right\}+\gamma^{\prime}\left(q_{j}\right)\left|\hat{x}^{\top}\right|^{2} \mathrm{~d} \mu_{T}=\int_{E} \gamma^{\prime}\left(q_{j}\right)\left|\hat{x}^{\top}\right|^{2} \mathrm{~d} \mu_{T} .
$$

Now notice that $\left|\hat{x}^{\top}\right|^{2}=0$ iff $\hat{x} \perp \mathcal{T}_{x} M$, this means in particular $x_{n+k-j+1}=$ $\cdots=x_{n+k}=0$ in $E$. In other words, if $x_{n+k-j+l} \neq 0$ for (at least) some $l=$ $1, \ldots, j$, then $\left|\hat{x}^{\top}\right|^{2}>0$ in $E$. Again, because of $\gamma$, this establishes

spt $\mu_{T} \cap\left(E \cap\left\{x \in \mathbb{R}^{n+k}: x_{n+k-j+l} \neq 0\right.\right.$ for one $\left.\left.l=1, \ldots, j\right\}\right) \subset H_{j}(R+\varepsilon)$

and combining both results, we arrive at

$$
\text { spt } \mu_{T} \subset H_{j}(R+\varepsilon) \cup\left(E \cap\left\{x \in \mathbb{R}^{n+k}: x_{n+k-j+1}=0, \ldots, x_{n+k}=0\right\}\right) .
$$

Finally, we claim that $E \cap\left\{x \in \mathbb{R}^{n+k}: x_{n+k-j+1}=0, \ldots, x_{n+k}=0\right\} \subset$ $H_{j}(R+\varepsilon)$. Assume there exists $\tilde{x}=\left(\tilde{x}_{1}, \ldots, \tilde{x}_{n+k}\right) \in \operatorname{spt} \mu_{T}$ such that $\tilde{x} \in E$ and $\tilde{x}_{n+k-j+1}=\cdots=\tilde{x}_{n+k}=0$ but $\tilde{x} \notin H_{j}(R+\varepsilon)$. Choose a function $f \in$ $C_{c}^{0}\left(\mathbb{R}^{n+k}\right)$ which satisfies $f \equiv 0$ in $\left\{x \in \mathbb{R}^{n+k}: x_{n+k-j+1}=0, \ldots, x_{n+k}=0\right\}$ and is strict positive anywhere else in some sufficiently large ball. Then, since $\tilde{x}$ is off the closed hyperboloid together with the fact (2.1), we have that in a small neighborhood points of $\operatorname{spt} T$ fulfill $x_{n+k-j+1}=0, \ldots, x_{n+k}=0$. By definition of $f$, this means in particular

$$
\lim _{\lambda \searrow 0} \int_{\eta_{\tilde{x}, \lambda}(M)} f(y) \theta(\tilde{x}+\lambda y) \mathrm{d} \mathcal{H}^{n}(y)=0 .
$$

Notice that, because of $\tilde{x} \in E$, we have $e_{n+k-j+1}, \ldots, e_{n+k} \in \mathcal{T}_{\tilde{x}} M$. In other words, $\mathcal{T}_{\tilde{x}} M \not \subset\left\{x \in \mathbb{R}^{n+k}: x_{n+k-j+1}=0, \ldots, x_{n+k}=0\right\}$.

This leads to a contradiction to the definition of the approximate tangent space $\theta(\tilde{x}) \int_{\mathcal{T}_{\tilde{x} M}} f(y) \mathrm{d} \mathcal{H}^{n}(y)>0$. This gives spt $T=\operatorname{spt} \mu_{T} \subset H_{j}(R+\varepsilon)$ and since $\varepsilon>0$ was arbitrary, the theorem is established.

\subsection{Non-existence theorem.}

Definition 2.3. A current $T \in \mathcal{R}_{n}(U)$ is called connected if $\operatorname{spt} T$ is a connected set.

For $j=1$ and $R=0$, we get the cone $H_{1}(0)=K:=\left\{\left(x_{1}, \ldots, x_{n+k}\right) \in\right.$ $\left.\mathbb{R}^{n+k}: x_{1}^{2}+\cdots+x_{n+k-1}^{2} \leq(n-1) x_{n+k}^{2}\right\}$. Let $K^{ \pm}:=K \cap\left\{ \pm x_{n+k}>0\right\}$ and $T^{ \pm}:=T\left\llcorner\left\{x \in \mathbb{R}^{n+k}: \pm x_{n+k}>0\right\} \in \mathcal{R}_{n}\left(\mathbb{R}^{n+k}\right)\right.$. From Theorem 2.2, we deduce that spt $T^{+} \subset \bar{K}^{+}=K^{+} \cup\{0\}$ and $\operatorname{spt} T^{-} \subset \bar{K}^{-}=K^{-} \cup\{0\}$. 
Lemma 2.4. Let $T \in \mathcal{R}_{n}\left(\mathbb{R}^{n+k}\right)$ be a stationary current in $\mathbb{R}^{n+k}$ and $\operatorname{spt} T \subset$ $K$. Then both currents $T^{+} \in \mathcal{R}_{n}\left(\mathbb{R}^{n+k}\right)$ and $T^{-} \in \mathcal{R}_{n}\left(\mathbb{R}^{n+k}\right)$ are also stationary in $\mathbb{R}^{n+k}$.

Proof. We consider $T^{+}$. Let $\varepsilon>0$ be arbitrary and $\phi_{\varepsilon}(t) \in C^{1}(\mathbb{R})$ be nonnegative such that $\phi_{\varepsilon}(t) \equiv 0, t \leq 0$, and $\phi_{\varepsilon}(t) \equiv 1, t>\varepsilon$, as well as $0 \leq \phi_{\varepsilon}^{\prime}(t) \leq c / \varepsilon$ for all $t \in \mathbb{R}$ for some $c>0$. Because $K^{+}$is contained in $\left\{x \in \mathbb{R}^{n+k}: x_{n+k}>0\right\}$, it follows $K^{+} \cap\left\{x \in \mathbb{R}^{n+k}: x_{n+k}<\varepsilon\right\} \subset B_{c \varepsilon}(0)$ for some (other) $c>0$, which depends only on the angle of the cone, i.e. on the dimension $n$. From monotonicity for stationary currents, the estimate $\mu_{T}\left(B_{c \varepsilon}(0)\right) \leq c \varepsilon^{n}$ holds true for small $\varepsilon>0$. Let $X$ be an arbitrary compactly supported $C^{1}$ vector field in $\mathbb{R}^{n+k}$ and spt $X \cap \operatorname{spt} \partial T^{+}=\emptyset$. In view of the stationarity of $T$, we can test $\phi_{\varepsilon}\left(x_{n+k}\right) X$. Thus

$$
\begin{aligned}
0 & =\int_{K^{+} \cap\left\{x_{n+k}<\varepsilon\right\}} \phi_{\varepsilon}^{\prime}\left(x_{n+k}\right)\left\langle e_{n+k}^{\top}, X\right\rangle \mathrm{d} \mu_{T}+\int_{\mathbb{R}^{n+k}} \phi_{\varepsilon}\left(x_{n+k}\right) \operatorname{div} M X \mathrm{~d} \mu_{T} \\
& \leq \frac{c}{\varepsilon}\left|e_{n+k}^{\top}\right| \sup _{\mathbb{R}^{n+k}}|X| \int_{B_{c \varepsilon}(0)} \mathrm{d} \mu_{T}+\int_{\mathbb{R}^{n+k}} \phi_{\varepsilon}\left(x_{n+k}\right) \operatorname{div}_{M} X \mathrm{~d} \mu_{T} \\
& \leq \frac{c}{\varepsilon} \mu_{T}\left(B_{c \varepsilon}(0)\right)+\int_{\mathbb{R}^{n+k}} \phi_{\varepsilon}\left(x_{n+k}\right) \operatorname{div}_{M} X \mathrm{~d} \mu_{T} \\
& \leq c \varepsilon^{n-1}+\int_{\mathbb{R}^{n+k}} \phi_{\varepsilon}\left(x_{n+k}\right) \operatorname{div}_{M} X \mathrm{~d} \mu_{T} \\
& \stackrel{\varepsilon \searrow 0}{\longrightarrow} \int_{\mathbb{R}^{n+k}} \chi_{\left\{x_{n+k} \geq 0\right\}} \operatorname{div}_{M} X \mathrm{~d} \mu_{T}=\int_{\mathbb{R}^{n+k}} \operatorname{div}_{M} X \mathrm{~d} \mu_{T^{+}} .
\end{aligned}
$$

The same inequality holds true for $-X$ and establishes the stationarity of $T^{+}$.

Applying Theorem 2.1 to $T^{+}$and $T^{-}$, respectively, together with the fact that spt $\partial T^{ \pm} \subset K^{ \pm}$, we get $0 \notin \operatorname{spt} T$. Since all calculations are invariant under translations, we have proved the following theorem.

Theorem 2.5 (Non-existence theorem). Let $\mathcal{K}=\mathcal{K}^{+} \cup\left\{p_{0}\right\} \cup \mathcal{K}^{-} \subset \mathbb{R}^{n+k}$ be a cone with vertex $p_{0}$ which is congruent to $K$. Then there exists no stationary current $T \in \mathcal{R}_{n}\left(\mathbb{R}^{n+k}\right)$ such that $\mathrm{spt} T$ is compact and connected with boundary values spt $\partial T \subset \mathcal{K}^{+} \cup \mathcal{K}^{-}$such that both spt $\partial T \cap \mathcal{K}^{+}$and $\operatorname{spt} \partial T \cap \mathcal{K}^{-}$are non-empty.

Remark. As $n$ increases, the angle of aperture $\beta$ of $K$ is increasing as well. Precisely we have $\beta=\arctan (\sqrt{n-1})$ and therefore $\beta \rightarrow 90^{\circ}$ as $n \rightarrow \infty$. The enclosure and non-existence theorems extend the existence theorem for currents with integer multiplicity, cf. [15, Lem. 34.1], as we get more information of the solution, e.g., the shape or the disconnectedness in at least two parts.

The proof of the following is the same as in [3, Cor.3]. 
Corollary 2.6 (Necessary conditions). Let $B_{1}, B_{2} \subset \mathbb{R}^{n+k}$ be two closed sets and suppose there exists a connected, stationary current $T \in \mathcal{R}_{n}\left(\mathbb{R}^{n+k}\right)$ in $\mathbb{R}^{n+k}$ with spt $\partial T \subset B_{1} \cup B_{2}$ and that both $\operatorname{spt} \partial T \cap B_{1} \neq \emptyset$ and $\operatorname{spt} \partial T \cap B_{2} \neq \emptyset$. Then we have:

(i) If $B_{i}:=\left\{x \in \mathbb{R}^{n+k}:\left|x-y_{i}\right| \leq \delta_{i}\right\}, i=1,2$, are closed balls with centers $y_{i}$ and radii $\delta_{i}$ and $R:=\left|y_{1}-y_{2}\right|$, then $R \leq\left(\frac{n}{n-1}\right)^{1 / 2}\left(\delta_{1}+\delta_{2}\right)$.

(ii) If $B_{1}$ and $B_{2}$ are arbitrary compact sets of diameter $d_{1}$ and $d_{2}$, separated by a slab of width $r>0$, then $r \leq \frac{1}{2}\left(\frac{2 n(n+k)}{(n-1)(n+k-1)}\right)^{1 / 2}\left(d_{1}+d_{2}\right)$.

2.3. Optimal results in codimension one. The question arises if it is possible to "enlarge" the cone and still prove non-existence. We restrict ourselves to codimension $k=1$. We use Dierkes' [3] construction of $n$-dimensional catenoids enclosing a cone with a larger angle of aperture. Therefore we show that the enclosing procedure also holds true for currents.

Consider a curve $(x, y(x))$ in $\mathbb{R}^{2}$ and its rotational symmetric $n$-dimensional graph $\left\{(x, y(x) \omega) \in \mathbb{R} \times \mathbb{R}^{n}: x \in\left[x_{0}, x_{1}\right], \omega \in \mathcal{S}^{n-1}\right\}$. Its area is proportional to the one dimensional integral $\int_{x_{0}}^{x_{1}} y^{n-1}(x) \sqrt{1+y^{\prime}(x)^{2}} \mathrm{~d} x$. Stationary solutions of this variational integral correspond to $n$-dimensional minimal submanifolds in $\mathbb{R}^{n+1}$. Results from calculus of variation give for a solution $y(x)$, the family of inverse functions $x(y)=a \int_{n-1}^{y} \sqrt{a} \frac{1}{\sqrt{\xi^{2(n-1)}-a^{2}}} \mathrm{~d} \xi+c$ for all $a>0, c \in \mathbb{R}$, and for all $y \geq \sqrt[n-1]{a}$. Then every member of the one-parameter family $x=$ $g(y, a), a>0$, is tangent to the half line $y=\tau_{0} x, x>0$ (independent of $a$ ), where $\tau_{0}:=\left(z_{0}^{2(n-1)}-1\right)^{1 / 2}$ and $z_{0}$ is the unique solution of

$$
\frac{z}{\sqrt{z^{2(n-1)}-1}}=\int_{1}^{z} \frac{\mathrm{d} \xi}{\sqrt{\xi^{2(n-1)}-1}} .
$$

Furthermore every point of the half line is contained in exactly one member of this family.

Let $f(\cdot, a)$ be the family of inverse functions and extend $f$ by even reflection, $f(x, a)=f(-x, a)$ for $x \leq 0$, then we have a smooth function $f(\cdot, a): \mathbb{R} \rightarrow \mathbb{R}$. Define $\rho:=\left(x_{1}^{2}+\cdots+x_{n}^{2}\right)^{1 / 2}$, then $\mathcal{M}_{a}:=\left\{x \in \mathbb{R}^{n+1}: \rho=f\left(x_{n+1}, a\right)\right\}, a>0$, is a family of smooth $n$-dimensional minimal submanifolds in $\mathbb{R}^{n+1}$. Furthermore $C_{a}:=\left\{x \in \mathbb{R}^{n+1}: \rho \leq f\left(x_{n+1}, a\right)\right\}, a>0$, "encloses" by construction the cone $K^{*}:=K \cup\{0\}:=\left\{x \in \mathbb{R}^{n+1}: \rho< \pm \tau_{0} x_{n+1}\right\} \cup\{0\}$.

Proposition 2.7 (Enclosure result.) Let $T \in \mathcal{R}_{n}\left(\mathbb{R}^{n+1}\right)$ be a stationary current in $\mathbb{R}^{n+1}$ with compact support and spt $\partial T \subset K=\left\{x \in \mathbb{R}^{n+1}: \rho< \pm \tau_{0} x_{n+1}\right\}$. Then we have spt $T \subset \bar{K}$.

Proof. We will show spt $T \subset C_{a}$ for all $a>0$. Because of $\bigcap_{a>0} C_{a}=\bar{K}$, the proposition is then established. Let us assume spt $T \not \subset C_{a}$ for some $a>0$ and consider $\eta_{0, \lambda \#} T \in \mathcal{R}_{n}\left(\mathbb{R}^{n+1}\right)$ with minimal $\lambda>1$ s.t. $\operatorname{spt}\left(\eta_{0, \lambda \#} T\right) \subset C_{a}$. Then there exists $p \in \operatorname{spt}\left(\eta_{0, \lambda \#} T\right)$ with $p \in \partial C_{a}$. The contracted current lies completely on one side of the smooth submanifold $\partial C_{a}$ and touches it at least 
in $p$. We want to apply the maximum principle of Solomon and White [16]. Therefore let us abbreviate for the fixed $\lambda: \eta(x):=\eta_{0, \lambda}(x)$ and $\tilde{T}:=\eta_{0, \lambda \#} T=$ $\eta_{\#} T$.

\section{Claims:}

(i) If spt $\partial T \subset K$, then we have spt $\partial \tilde{T} \subset K$.

(ii) $\mathcal{T}_{x} M$ exists in $x$ iff $\mathcal{T}_{\eta(x)} \eta(M)$ exists in $\eta(x)$ and we have equality.

(iii) Let $T$ be stationary in $\mathbb{R}^{n+1}$, then so is $\tilde{T}=\tau\left(\eta(M), \theta_{\tilde{T}}, \xi_{\tilde{T}}\right)$.

Claim (i) and (ii) are proved by direct calculations. For the third statement, note that we have for the Jacobian, $J_{M} \eta=\lambda^{-n}$, cf. [15, Ch. 12]. Let $Y \in$ $C_{c}^{1}\left(\mathbb{R}^{n+1} \backslash \operatorname{spt}\left(\partial\left(\eta_{0, \lambda \#} T\right)\right), \mathbb{R}^{n+1}\right)$ be arbitrary. Then $X(x):=Y \circ \eta(x)$ is of class $C_{c}^{1}\left(\mathbb{R}^{n+1} \backslash \operatorname{spt}(\partial T), \mathbb{R}^{n+1}\right)$. We calculate the variation of the contracted current

$$
\begin{aligned}
\delta \tilde{T}(Y) & =\int_{\mathbb{R}^{n+1}} \operatorname{div}_{\eta(M)} Y(y) \mathrm{d} \mu_{\tilde{T}}(y) \\
& =\int_{\eta(M)} \operatorname{div}_{\eta(M)} Y(y) \theta_{\tilde{T}}(y) \mathrm{d} \mathcal{H}^{n}(y) \\
& =\int_{\eta(M)} \operatorname{div}_{\eta(M)}\left(X \circ \eta^{-1}(y)\right) \theta_{T} \circ \eta^{-1}(y) \mathrm{d} \mathcal{H}^{n}(y) .
\end{aligned}
$$

We have for the first factor

$$
\begin{aligned}
\operatorname{div}_{\eta(M)}\left(X \circ \eta^{-1}(y)\right) & =\sum_{i=1}^{n+1}\left\langle\mathcal{P}_{\mathcal{T}_{y} \eta(M)}\left[D\left(X_{i} \circ \eta^{-1}(y)\right)\right], e_{i}\right\rangle \\
& =\sum_{i=1}^{n+1}\left\langle\mathcal{P}_{\mathcal{T}_{y} \eta(M)}\left[D X_{i} \circ \eta^{-1}(y) \mathbb{I}_{n+1} \lambda\right], e_{i}\right\rangle \\
& =\lambda \sum_{i=1}^{n+1}\left\langle\mathcal{P}_{\mathcal{T}_{\eta(x)} \eta(M)}\left[D X_{i} \circ \eta^{-1}(y)\right], e_{i}\right\rangle \\
& =\lambda \sum_{i=1}^{n+1}\left\langle\mathcal{P}_{\mathcal{T}_{\eta(x)} \eta(M)}\left[D X_{i}\right], e_{i}\right\rangle \circ \eta^{-1}(y) \\
& =\lambda\left(\operatorname{div}_{M} X\right) \circ \eta^{-1}(y)
\end{aligned}
$$

where we have used the second claim. Finally, we get by applying the area formula

$$
\begin{aligned}
& \delta \tilde{T}(Y)=\lambda \underset{\eta(M)}{\int}\left(\operatorname{div}_{M} X \theta_{T}\right) \circ \eta^{-1} \mathrm{~d} \mathcal{H}^{n}=\lambda \int_{M} \operatorname{div}_{M} X \theta_{T} J_{M} \eta \mathrm{d} \mathcal{H}^{n} \\
& =\lambda \lambda^{-n} \int_{M} \operatorname{div}_{M} X \theta_{T} \mathrm{~d} \mathcal{H}^{n}=\lambda^{-n+1} \int_{M} \operatorname{div}_{M} X \mathrm{~d} \mu_{T}=0 .
\end{aligned}
$$

Thus $\tilde{T}$ is stationary in $\mathbb{R}^{n+1}$ because $Y$ was arbitrary. Now we are able to apply the maximum principle [16] since the support of $T$ is compact. Therefore 
we get coincidence of spt $\tilde{T}$ and $\partial C_{a}$ in an open subset. This is a contradiction to claim (i).

With exactly the same argument as in Section 2.2, we can prove the nonexistence theorem. Furthermore it is optimal because the cone is enclosed by a family of minimal submanifolds.

Theorem 2.8 (Optimal non-existence theorem). Let $\mathcal{K}=\mathcal{K}^{+} \cup\left\{p_{0}\right\} \cup \mathcal{K}^{-} \subset$ $\mathbb{R}^{n+1}$ be a cone with vertex $p_{0}$ congruent to $K^{ \pm} \cup\{0\}=\left\{x \in \mathbb{R}^{n+1}: x_{1}^{2}+\right.$ $\left.\cdots+x_{n}^{2}-\tau_{0}^{2} x_{n+1}^{2}<0, \pm x_{n+1}>0\right\} \cup\{0\}$. Then there is no stationary current $T \in \mathcal{R}_{n}\left(\mathbb{R}^{n+1}\right)$ with compact support such that $\operatorname{spt} T$ is connected and the boundary fulfills spt $\partial T \subset \mathcal{K}^{+} \cup \mathcal{K}^{-}$such that $\mathrm{spt} \partial T \cap \mathcal{K}^{+}$as well as spt $\partial T \cap \mathcal{K}^{-}$ is non-empty.

\section{Currents with mean curvature vector.}

3.1. Enclosure theorem. The enclosure Theorem 2.2 naturally extends to currents with mean curvature vector $\mathbf{H} \in L_{\text {loc }}^{1}\left(\mu_{T}\right)$ under appropriate conditions. To this end, we define the quadratic function $q_{j}(x)$ with an additional parameter $b \in[0,1]$. Thus $q_{j}(x):=r_{j}(x)-(n-j) / j b s_{j}(x)$ and again $H_{j}(R):=\{x \in$ $\left.\mathbb{R}^{n+k}: q_{j}(x) \leq R\right\}$.

Theorem 3.1 (Enclosure theorem). Let $T \in \mathcal{R}_{n}\left(\mathbb{R}^{n+k}\right)$ be a current in $\mathbb{R}^{n+k}$ with mean curvature vector $\mathbf{H}(x)$ and compact support spt $T$. It satisfies spt $\partial T$ $\subset H_{j}(R)$ for $R \in \mathbb{R}, j=1, \ldots, n-1$, and $b \in[0,1]$. The mean curvature vector $\mathbf{H}$ fulfills

$$
b+|\mathbf{H}(x)|\left[\frac{r_{j}(x)}{(n-j)^{2}}+\frac{b^{2}}{j^{2}} s_{j}(x)\right]^{1 / 2} \leq 1 \text { for } \mu_{T}-\text { a.e. } x \in \mathbb{R}^{n+k} \backslash H_{j}(R) .
$$

Then $\operatorname{spt} T \subset H_{j}(R)$.

Proof. The proof is similar to the case $\mathbf{H} \equiv 0$. Define

$$
\hat{x}(x):=\left(x_{1}, \ldots, x_{n+k-j},-\frac{n-j}{j} b x_{n+k-j+1}, \ldots,-\frac{n-j}{j} b x_{n+k}\right)
$$

and consider $X(x):=\Psi_{\text {spt } T}(x) \gamma\left(q_{j}(x)\right) \hat{x}(x)$. Note that the additional term can be estimated directly with the Cauchy-Schwarz inequality $\langle\mathbf{H}(x)$, $\hat{x}(x)\rangle \geq-|\mathbf{H}(x)||\hat{x}(x)|=-|\mathbf{H}(x)|\left[r_{j}(x)+\left(\frac{n-j}{j}\right)^{2} b^{2} s_{j}(x)\right]^{1 / 2}$. Plugging all terms into (1.1) gives

$$
\begin{aligned}
0 & =\int_{\mathbb{R}^{n+k}}\left\langle\nabla_{M} \gamma\left(q_{j}\right), \hat{x}\right\rangle+\operatorname{div}_{M} \hat{x} \gamma\left(q_{j}\right)+\gamma\left(q_{j}\right)\langle\hat{x}, \mathbf{H}\rangle \mathrm{d} \mu_{T} \\
& \geq \int_{\mathbb{R}^{n+k}} \gamma\left(q_{j}\right)\left\{n-\left(1+\frac{n-j}{j} b\right) \sum_{i=n+k-j+1}^{n+k} p_{i i}-|\mathbf{H}|\left[r_{j}+\left(\frac{n-j}{j}\right)^{2} b^{2} s_{j}\right]^{1 / 2}\right\} \\
& \quad+2 \gamma^{\prime}\left(q_{j}\right)\left|\hat{x}^{\top}\right|^{2} \mathrm{~d} \mu_{T} .
\end{aligned}
$$


We can estimate the expression $\{\ldots\}$ pointwise

$$
\begin{aligned}
n & -\left(1+\frac{n-j}{j} b\right) \sum_{i=n+k-j+1}^{n+k} p_{i i}-|\mathbf{H}|\left[r_{j}+\left(\frac{n-j}{j}\right)^{2} b^{2} s_{j}\right]^{1 / 2} \\
& \geq n-\left(1+\frac{n-j}{j} b\right) j-|\mathbf{H}|\left[r_{j}+\left(\frac{n-j}{j}\right)^{2} b^{2} s_{j}\right]^{1 / 2} \\
& =(n-j)\left((1-b)-|\mathbf{H}|\left[\frac{r_{j}}{(n-j)^{2}}+\left(\frac{b}{j}\right)^{2} s_{j}\right]^{1 / 2}\right)
\end{aligned}
$$

and now notice that the resulting term is non-negative in $\mathbb{R}^{n+k} \backslash H_{j}(R)$ for $\mu_{T}$-a.e. $x$. Next we integrate over the sets $E$ and $\mathbb{R}^{n+k} \backslash E$ where $E$ is defined exactly as above. In the latter case, we evidently have strict inequality in (3.2) as not all $p_{i i}$ are equal to one. Thus spt $\mu_{T} \cap\left(\mathbb{R}^{n+k} \backslash E\right) \subset H_{j}(R)$. The proof is now finished as before.

We mention two sufficient characterizations for the mean curvature vector $\mathbf{H}$ such that the condition (3.1) is satisfied. Both are easier to check.

Lemma 3.2. The condition (3.1) is fulfilled provided

(i) $q:=|x||\mathbf{H}(x)|<1$ for $\mu_{T}$-a.e. $x \in \mathbb{R}^{n+k} \backslash H_{j}(R)$ and $b \leq 1-q$ or

(ii) $q:=|x||\mathbf{H}(x)|<n-j$ for $\mu_{T}$-a.e. $x \in \mathbb{R}^{n+k} \backslash H_{j}(R)$ and $b \leq \min \left\{\frac{1}{n-1}, 1-\right.$ $\left.\frac{q}{n-j}\right\}$.

Proof. A calculation gives

$$
b+|\mathbf{H}|\left[\frac{r_{j}(x)}{(n-j)^{2}}+\frac{b^{2}}{j^{2}} s_{j}(x)\right]^{1 / 2} \leq b+|\mathbf{H}||x|=b+q \leq 1
$$

thus i) is established. Estimations of $b$ yield the second claim

$$
\begin{aligned}
b+ & |\mathbf{H}|\left[\frac{r_{j}(x)}{(n-j)^{2}}+\frac{b^{2}}{j^{2}} s_{j}(x)\right]^{1 / 2}=b+|\mathbf{H}| \frac{1}{n-j}\left[r_{j}(x)+\left(\frac{n-j}{j}\right)^{2} b^{2} s_{j}(x)\right]^{1 / 2} \\
& \leq 1-\frac{1}{n-j} q+|\mathbf{H}| \frac{1}{n-j}\left[r_{j}(x)+\left(\frac{n-j}{j}\right)^{2}\left(\frac{1}{n-1}\right)^{2} s_{j}(x)\right]^{1 / 2} \\
& \leq 1-\frac{1}{n-j} q+|\mathbf{H}| \frac{1}{n-j}|x|=1-\frac{1}{n-j} q+\frac{1}{n-j} q=1 .
\end{aligned}
$$

3.2. Non-existence theorem. Now let $T \in \mathcal{R}_{n}\left(\mathbb{R}^{n+k}\right)$ be a current with $0 \in$ spt $T \subset K:=H_{1}(0)$. Notice, for any current $T=\tau(M, \theta, \xi)$, we have an associated rectifiable varifold $V=v(M, \theta)$ just by dropping the orientation. In fact, we have as in [15].

Proposition 3.3. Let $V$ be a rectifiable varifold in some neighborhood of the origin. Suppose the density exists in 0 and for $V_{j}:=\eta_{0, \lambda_{j} \#} V, \lambda_{j} \searrow 0$, we have $\mu_{V_{j}} \rightarrow \mu_{C}$ in $\mathbb{R}^{n+k}$ such that $\mu_{C}$ is associated to a rectifiable varifold $C$ which is stationary in $\mathbb{R}^{n+k}$. Then $C$ is a cone. 
Theorem 3.4. Let $V=v(M, \theta)$ and $C=v(N, \vartheta)$ be two varifolds as above. If spt $V \subset K:=\left\{x \in \mathbb{R}^{n+k}: x_{1}^{2}+\cdots+x_{n+k-1}^{2} \leq(n-1) b x_{n+k}^{2}\right\}$, then we have spt $C \subset K$ as well.

Lemma 3.5. Let $\left(\mu_{j}\right)_{j \in \mathbb{N}}, \mu$ be Radon measures with $\mu_{j} \rightarrow \mu, j \rightarrow \infty$, in $\mathbb{R}^{n+k}$ (weak convergence of Radon measures). Assume for some closed set $A$, we have spt $\mu_{j} \subset A$ for all $j \in \mathbb{N}$. Then spt $\mu \subset A$.

Proof. We show $x \notin A$ implies the existence of a $\rho>0$ such that $\mu\left(B_{\rho}(x)\right)=$ 0 . From the hypothesis, we have $\operatorname{dist}(x, A)>\varepsilon>0$ and therefore define $\rho:=\frac{1}{3} \operatorname{dist}(x, A)$. Notice, because of $\operatorname{spt} \mu_{j} \subset A$ for all $j \in \mathbb{N}$, it evidently follows $B_{2 \rho}(x) \cap \operatorname{spt} \mu_{j}=\emptyset$ for all $j \in \mathbb{N}$. Now choose a continuous function $\phi: \mathbb{R}^{n+k} \rightarrow[0,1]$ satisfying $\left.\phi\right|_{B_{\rho}(x)} \equiv 1$ and $\left.\phi\right|_{\mathbb{R}^{n+k} \backslash B_{2 \rho}(x)} \equiv 0$. Since

$0 \leq \mu\left(B_{\rho}(x)\right) \leq \liminf _{j \rightarrow \infty} \mu_{j}\left(B_{\rho}(x)\right) \leq \liminf _{j \rightarrow \infty} \int_{\mathbb{R}^{n+k}} \phi \mathrm{d} \mu_{j}=\liminf _{j \rightarrow \infty} \int_{\text {spt } \mu_{j}} \phi \mathrm{d} \mu_{j}=0$,

this completes the proof.

Proof of Theorem 3.4. Again, define $V_{j}:=\eta_{0, \lambda_{j} \#} V$, then we know for every $j$ that $\operatorname{spt} V_{j}=\operatorname{spt}\left(\eta_{0, \lambda_{j} \#} V\right) \subset \eta_{0, \lambda_{j}}(\operatorname{spt}(V)) \subset \eta_{0, \lambda_{j}}(K)=K$. From Proposition 3.3 , we obtain $\mu_{V_{j}} \rightarrow \mu_{C}, j \rightarrow \infty$, and Lemma 3.5 gives spt $C=\operatorname{spt} \mu_{C} \subset$ $K$ as required.

We consider the two restricted varifolds $C^{ \pm}:=C\left\llcorner\left\{x: \pm x_{n+k}>0\right\}=\right.$ $v\left(N \cap\left\{x: \pm x_{n+k}>0\right\},\left.\vartheta\right|_{N \cap\left\{x: \pm x_{n+k}>0\right\}}\right)$ satisfying spt $C^{+} \subset \bar{K}^{+}=K^{+} \cup$ $\{0\} \subset\left\{x \in \mathbb{R}^{n+k}: x_{n+k} \geq 0\right\}$ and spt $C^{-}$is obviously contained in $\{x \in$ $\left.\mathbb{R}^{n+k}: x_{n+k} \leq 0\right\}$. Both varifolds are still cones and literally as in the proof of Lemma 2.4, it follows that both $C^{+}$and $C^{-}$are stationary in $\mathbb{R}^{n+k}$. Hence we can apply the following lemma to conclude a contradiction. For a proof, see Simon [15, Thm. 36.5 \& Rmk. 36.6].

Lemma 3.6. Let $C=v(N, \vartheta)$ be a rectifiable varifold stationary in $\mathbb{R}^{n+k}$ and satisfying $\eta_{0, \lambda \#} C=C$ for all $\lambda>0$. Furthermore assume $\operatorname{spt} C \subset \overline{\mathfrak{H}}$ where $\mathfrak{H}$ is some open half space in $\mathbb{R}^{n+k}$ with $0 \in \partial \mathfrak{H}$. Then $\operatorname{spt} C \subset \partial \mathfrak{H}$.

This gives spt $C^{ \pm} \subset\left\{x_{n+k}=0\right\}$ in contradiction to $\operatorname{spt} C^{ \pm} \subset \bar{K}=K$ because by construction evidently $C \not \equiv 0$. Notice in particular this means that the support of a stationary tangent cone cannot pass through the vertex of the cone $K$ and therefore the origin cannot be contained in the support of the current $T$. In order to fulfill the assumptions of Proposition 3.3, we assume $\mathbf{H} \in L_{\mathrm{loc}}^{p}\left(U, \mathbb{R}^{n+k} ; \mu_{T}\right)$ for some $p>n$ and $\theta \geq 1 \mu_{T}$-a.e. in $U$ for some small neighborhood $U$ of the origin with $U \cap \operatorname{spt} \partial T=\emptyset$. This gives the existence of an area stationary tangent cone in every $x \in \operatorname{spt} T \cap U$, see [15, Ch. 8]. Finally, this establishes

Theorem 3.7 (Non-existence theorem). Let $T \in \mathcal{R}_{n}\left(\mathbb{R}^{n+k}\right)$ be a current with compact support $\operatorname{spt} T$ and mean curvature vector $\mathbf{H}$. Assume for some $b \in$ $[0,1]$,

$$
\operatorname{spt} \partial T \subset K^{ \pm}:=\left\{x \in \mathbb{R}^{n+k}: \sum_{i=1}^{n+k-1} x_{i}^{2} \leq(n-1) b x_{n+k}^{2}, \pm x_{n+k}>0\right\}
$$


such that both spt $\partial T \cap K^{+}$and spt $\partial T \cap K^{-}$are non-empty. Furthermore define $r_{1}(x):=x_{1}^{2}+\cdots+x_{n+k-1}^{2}$ and suppose the mean curvature vector $\mathbf{H}$ satisfies on the one hand

$$
b+|\mathbf{H}|\left[\frac{r_{1}(x)}{(n-1)^{2}}+b^{2} x_{n+k}^{2}\right]^{1 / 2} \leq 1 \text { for } \mu_{T}-\text { a.e. } x \in \mathbb{R}^{n+k} \backslash K
$$

and on the other hand, in some neighborhood $U$ of the origin, $\mathbf{H} \in L_{\text {loc }}^{p}\left(U, \mathbb{R}^{n+k}\right.$; $\left.\mu_{T}\right)$ for some $p>n$ and $\theta(x) \geq 1$ for $\mu_{T}$-a.e. $x \in U$. Then $\operatorname{spt} T$ cannot be connected.

Remark. Instead of condition (3.3), one of the requirements of Lemma 3.2 with $j=1$ and $R=0$ can also be fulfilled. The condition $\theta \geq 1 \mu_{T}$-a.e. is automatically given for currents with integer multiplicity.

4. Enclosure and non-existence theorems for currents in submanifolds. Here we discuss an important modification of stationarity in Euclidean spaces. More generally we let $\mathcal{N}$ be an $(n+l)$-dimensional $C^{2}$-submanifold of $\mathbb{R}^{n+k}$ for $0 \leq l \leq k$. We denote by $B_{y}: \mathcal{T}_{y} \mathcal{N} \times \mathcal{T}_{y} \mathcal{N} \rightarrow\left(\mathcal{T}_{y} \mathcal{N}\right)^{\perp}$ the second fundamental form of $\mathcal{N}$ at $y$. Then $T$ is called stationary in $\mathcal{N}$ if the first variational formula holds true with $\mathbf{H}_{M}:=\sum_{i=1}^{n} B_{x}\left(\tau_{i}, \tau_{i}\right)=\left.\operatorname{tr}\left(B_{x}\right)\right|_{\mathcal{T}_{x} M}$ and $\left\{\tau_{1}, \ldots, \tau_{n}\right\}$ is any orthonormal basis for the approximate tangent space $\mathcal{T}_{x} M$ of $T$ in $x$, cf. Simon [15, Def. 16.4].

Theorem 4.1 (General enclosure and non-existence theorem). Let $\mathcal{N}$ be an $(n+l)$-dimensional embedded $C^{2}$-submanifold of $\mathbb{R}^{n+k}$ for $0 \leq l \leq k$. Let $T=\tau(M, \theta, \xi) \in \mathcal{R}_{n}\left(\mathbb{R}^{n+k}\right), M \subset \mathcal{N}$ be a stationary current in $\mathcal{N}$ with compact support. Furthermore for some $R \in \mathbb{R}$, assume

$$
b+\left|\mathbf{H}_{M}(x)\right|\left[\frac{r_{j}(x)}{(n-j)^{2}}+\frac{b^{2}}{j^{2}} s_{j}(x)\right]^{1 / 2} \leq 1 \text { for } \mu_{T}-\text { a.e. } x \in \mathcal{N} \backslash H_{j}(R) .
$$

If $\operatorname{spt} \partial T \subset H_{j}(R)$, then $\operatorname{spt} T \subset H_{j}(R)$.

Let (4.1) be true for $j=1$ and $R=0$ and assume for $\varepsilon>0$, on the one hand, $\theta \geq 1 \mu_{T}$-a.e. in $B_{\varepsilon}(0)$ and on the other hand, $\mathbf{H}_{M} \in L_{\mathrm{loc}}^{p}\left(B_{\varepsilon}(0)\right.$, $\left.\mathbb{R}^{n+k} ; \mu_{T}\right)$ for some $p>n$. Furthermore let spt $\partial T \subset K^{ \pm}$such that both spt $\partial T \cap K^{+} \neq \emptyset$ and $\operatorname{spt} \partial T \cap K^{-} \neq \emptyset$. Then $\operatorname{spt} T$ cannot be a connected set.

We want to estimate the abstract curvature $\mathbf{H}_{M}$ against some quantity depending only on the submanifold $\mathcal{N}$. We restrict ourselves to the case $l=$ $k-1$. As usual, we can define the principal curvatures $\kappa_{1}, \ldots, \kappa_{n+k-1}$ of $\mathcal{N}$ which we order with respect to their absolute value: $\left|\kappa_{1}\right| \geq \cdots \geq\left|\kappa_{n+k-1}\right|$. We define $\Lambda_{n}:=\left|\kappa_{1}\right|+\cdots+\left|\kappa_{n}\right|$ what we call the $n$-mean curvature of $\mathcal{N}$. This number slightly differs from the usual $n$-mean curvature used in barrier principles of higher codimension, cf. $[6,7]$. We are now able to proceed similarly as in [12] and arrive at the important estimate: For $\mu_{T}$-a.e. $x \in M$, we have $\left|\mathbf{H}_{M}(x)\right|=\left|\operatorname{tr}\left(B_{x}\right)\right| \mathcal{T}_{x} M \mid \leq \Lambda_{n}(x)$. This is just a conclusion of the following general result where we skip the proof. 
Lemma 4.2. Let $Q: \mathfrak{V} \rightarrow \mathbb{R}$ be a quadratic form of an $N$-dimensional Euclidean vector space $\mathfrak{V}$ with ordered eigenvalues $\left|\kappa_{1}\right| \geq \cdots \geq\left|\kappa_{N}\right|$. Then we have for every $n$-dimensional subspace $\mathfrak{W} \subset \mathfrak{V}$, the estimate $|\operatorname{tr}(Q)|_{\mathfrak{W}}|\leq| \kappa_{1} \mid+\cdots+$ $\left|\kappa_{n}\right|$.

So we get results under conditions just depending on the ambient space.

Corollary 4.3 (Enclosure and non-existence theorem in hypersurfaces). Let $\mathcal{N}$ be an $(n+k-1)$-dimensional embedded $C^{2}$-submanifold of $\mathbb{R}^{n+k}$. Let $T=$ $\tau(M, \theta, \xi) \in \mathcal{R}_{n}\left(\mathbb{R}^{n+k}\right), M \subset \mathcal{N}$ be a stationary current in $\mathcal{N}$ with compact support. Furthermore for some $R \in \mathbb{R}$, assume

$$
b+\Lambda_{n}(x)\left[\frac{r_{j}(x)}{(n-j)^{2}}+\frac{b^{2}}{j^{2}} s_{j}(x)\right]^{1 / 2} \leq 1 \text { for } \mu_{T}-\text { a.e. } x \in \mathcal{N} \backslash H_{j}(R) .
$$

If $\operatorname{spt} \partial T \subset H_{j}(R)$, then $\operatorname{spt} T \subset H_{j}(R)$.

Let (4.2) be true for $j=1$ and $R=0$ and assume for $\varepsilon>0$, on the one hand, $\theta \geq 1 \mu_{T}$-a.e. in $B_{\varepsilon}(0)$ and on the other hand, $\Lambda_{n} \in L_{\mathrm{loc}}^{p}\left(B_{\varepsilon}(0), \mathbb{R}^{n+k} ; \mu_{T}\right)$ for some $p>n$. Furthermore let spt $\partial T \subset K^{ \pm}$such that both $\operatorname{spt} \partial T \cap K^{+} \neq \emptyset$ and spt $\partial T \cap K^{-} \neq \emptyset$. Then spt $T$ cannot be a connected set.

Example 4.4 (Non-existence of stationary currents in spheres). Define the shifted sphere $\mathcal{S}=\mathcal{S}^{n+k-1}\left(x_{0}, R\right):=\left\{x \in \mathbb{R}^{n+k}:\left|x-x_{0}\right|=R\right\}$ with $0 \in \mathcal{S}$. Let $T=\tau(M, \theta, \xi) \in \mathcal{R}_{n}\left(\mathbb{R}^{n+k}\right)$ with integer multiplicity and $M \subset \mathcal{S}$ be a stationary current in $\mathcal{S}$. Assume spt $T$ is compact, $q:=\sup _{x \in \operatorname{spt} T}|x| \frac{n}{R}<1$, and suppose spt $\partial T \subset K:=\left\{x \in \mathbb{R}^{n+k}: x_{1}^{2}+\cdots+x_{n+k-1}^{2} \leq(n-1)(1-q) x_{n+k}^{2}\right\}$ such that both spt $\partial T \cap\left(K \cap\left\{x_{n+k}>0\right\}\right) \neq \emptyset$ and $\operatorname{spt} \partial T \cap\left(K \cap\left\{x_{n+k}<0\right\}\right) \neq \emptyset$. Then $T$ cannot be connected.

Acknowledgements. Open Access funding provided by Projekt DEAL. This paper is part of my dissertation [8] written under supervision of Prof. Dr. U. Dierkes. The project was supported by the Studienstiftung des deutschen Volkes and Stanford University, where parts of this paper have been worked out.

Open Access. This article is licensed under a Creative Commons Attribution 4.0 International License, which permits use, sharing, adaptation, distribution and reproduction in any medium or format, as long as you give appropriate credit to the original author(s) and the source, provide a link to the Creative Commons licence, and indicate if changes were made. The images or other third party material in this article are included in the article's Creative Commons licence, unless indicated otherwise in a credit line to the material. If material is not included in the article's Creative Commons licence and your intended use is not permitted by statutory regulation or exceeds the permitted use, you will need to obtain permission directly from the copyright holder. To view a copy of this licence, visit http://creativecommons.org/ licenses/by/4.0/.

Publisher's Note Springer Nature remains neutral with regard to jurisdictional claims in published maps and institutional affiliations. 


\section{References}

[1] Böhme, R., Hildebrandt, S., Tausch, E.: The two-dimensional analogue of the catenary. Pac. J. Math. 88, 247-278 (1980)

[2] Clarenz, U.: Enclosure theorems for extremals of elliptic parametric functionals. Calc. Var. Partial Differ. Equ. 15, 313-324 (2002)

[3] Dierkes, U.: Maximum principles and nonexistence results for minimal submanifolds. Manuscr. Math. 69, 203-218 (1990)

[4] Dierkes, U., Hildebrandt, S., Sauvigny, F.: Minimal Surfaces. Springer, New York (2010)

[5] Dierkes, U., Hildebrandt, S., Tromba, A.J.: Regularity of Minimal Surfaces. Springer, New York (2010)

[6] Dierkes, U., Schwab, D.: Maximum principles for submanifolds of arbitrary codimension and bounded mean curvature. Calc. Var. Partial Differ. Equ. 22, 173$184(2005)$

[7] Henkemeyer, P.: A barrier principle for surfaces with prescribed mean curvature and arbitrary codimension. Result. Math. 64, 67-75 (2013)

[8] Henkemeyer, P.: Einschließungs- und Nichtexistenzsätze in der geometrischen Maßtheorie. PhD thesis (2017)

[9] Henkemeyer, P.: Enclosure theorems and barrier principles for energy stationary currents and the associated Brakke-flow. Analysis (Berlin) 37(4), 223-241 (2017)

[10] Hildebrandt, S.: Maximum principles for minimal surfaces and for surfaces of continuous mean curvature. Math. Z. 128, 253-269 (1972)

[11] Ilmanen, T.: A strong maximum principle for singular minimal hypersurfaces. Calc. Var. Partial Differ. Equ. 4(5), 443-467 (1996)

[12] Jorge, L.P., Tomi, F.: The barrier principle for minimal submanifolds of arbitrary codimension. Ann. Glob. Anal. Geom. 24, 261-267 (2003)

[13] Osserman, R., Schiffer, M.: Doubly connected minimal surfaces. Arch. Rat. Mech. Anal. 58, 285-307 (1975)

[14] Schätzle, R.: Quadratic tilt-excess decay and strong maximum principle for varifolds. Ann. Sc. Norm. Super. Pisa Cl. Sci. (5) 3(1), 171-231 (2004)

[15] Simon, L.: Lectures on geometric measure theory. Australian National University, Proceedings of the Centre for Mathematical Analysis (1984)

[16] Solomon, B., White, B.: A strong maximum principle for varifolds that are stationary with respect to even parametric elliptic functionals. Indiana Univ. Math. J. 38, 683-691 (1989)

[17] White, B.: The maximum principle for minimal varieties of arbitrary codimension. Comm. Anal. Geom. 18(3), 421-432 (2010)

[18] Wickramasekera, N.: A sharp strong maximum principle and a sharp unique continuation theorem for singular minimal hypersurfaces. Calc. Var. Partial Differ. Equ. 51(3-4), 799-812 (2014)

[19] Winklmann, S.: Maximum principles for energy stationary hypersurfaces. Analysis (Munich) 26, 251-258 (2006) 
PATRick Henkemeyer

Fakultät für Mathematik

Universität Duisburg-Essen

45117 Essen

Germany

e-mail: patrick.henkemeyer@uni-due.de

Received: 12 January 2020 